\title{
THE METHOD OF MINIMAL NORMAL FORPFE
}

\author{
S.R. Mane and W.T. Weng \\ FEB i a w \\ Brookhaven National Laboratory, Upton, NY 11970 O S I
}

\section{ABSTRACT}

Normal form methods for solving nonlinear differential equations are reviewed and the comparative merits of three methods are evaluated. The concept of the minimal normal form is explained and is shown to be superior to other choices. The method is then extended to apply to the evaluation of discrete maps of an accelerator or otorage ring. Such an extension, as suggested in this paper, is more suited for accelerator-based applications than a formulation utilizing continuous differential equations. A computer code has been generated to systematically implement various normal form formulations for maps in two-dimensional phase space. Specific examples of quadratic and cubic nonlinear fields were used and solved by the method developed. The minimal normal form method shown here gives good results using relatively low order expansions.

\section{INTRODUCTION}

It is relatively easy and straightforward to find solutions of accelerator lattice design for a linear system [1]. Once the nonlinear elements are introduced, no preferred method has been found. Lately, one-turn maps [2] have been suggested as a useful tool for embodying all perturbations in an accelerator and hence a testing bed for evaluating the comparative merits of various approaches. A good one-turn map representation of an accelerator can provide all necessary information for the lattice description and hopefully also facilitate the evaluation of the long-term stability and dynamic aperture of the accelerator or storage ring.

A powerful techqniue of analyzing nonlinear one-turn maps is the use of normal forms. A recent theoretical development in this area is that of so-called minimal normal forms. Motivated by the successful and apparently superior performance of this method [3] in solving ordinary nonlinear differential equations, this report is a first attempt to apply the same method to the evaluation of maps suitable for accelerator design. A more detailed exposition can be found in Ref. [4]. A rapidly convergent method can provide basic reliable lattice information in a few terms, which is an important contribution in its own right. The larger payoff is in the trustworthiness of the prediction of long-term stability and accuracy in the the calculation of dynamic aperture of a given accelerator. A good general discussion of normal forms is given in Ref. [5], and an example of the application of normal form techniques, but not minimal normal forms, for the LHC (Large Hadron Collider) at CERN, is given in Ref. [6].

"Work performed under the auspices of the U.S. Department of Energy.

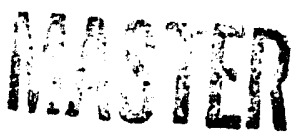




\section{REVIEW OF FORMALISM}

The minimal normal form method will be explained below. Many of the technical details can be found in Ref. [4]. First, differential equations will be treated. This will also serve the purpose of defining some notations and preparing the groundwork for an extension of the method to treat discrete maps. A description of the formalism, for differential equations, is also given in Ref. [3], which is not cast in a form directly useful to the development below.

Consider a dynamical system describable by a canonically conjugate (coordinate, momentum) pair $(x, p)$, with $z=x+i p$, which executes a harmonic oscillation with a nonlinear autonomous perturbation

$$
\dot{z}=-i \mu z+\sum_{k=1}^{\infty} \epsilon^{k} Z_{k}\left(z, z^{*}\right),
$$

where the frequency is $\mu, \epsilon$ is the small parameter of the perturbation expansion, and the $Z_{k}$ are homogenous polynomials of degree $k+1$ in $z$ and $z^{*}$, given by

$$
Z_{k}=\sum_{p+q=k+1} Z_{p q} z^{p} z^{* q}
$$

The above equation is solved by the use of a near-identity transformation to a normal form variable $u$ via

$$
z=u+\sum_{k=1}^{\infty} \epsilon^{k} T_{k}\left(u, u^{*}\right),
$$

with homogenous polynomials

$$
T_{k}=\sum_{p+q=k+1} T_{p q} u^{p} u^{* q}
$$

which are to be determined. The resulting equation of motion for $u$ is written in the form

$$
\dot{u}=-i \mu u+\sum_{k=1}^{\infty} \epsilon^{k} U_{k}\left(u, u^{*}\right),
$$

where the $U_{k}$ are also to be determined, and are obviously related to the $T_{k}$. The above equation is made into a normal form by choosing the functions $T_{k} 80$ that the $U_{k}$ contain resonant monomials only, i.e. monomials of the form $u\left(u u^{*}\right)^{p}, p=1,2, \ldots$, which means that $U_{k}=0$ for odd $k$, and

$$
\dot{u}=-i \mu u+\sum_{k} \epsilon^{2 k} \tilde{U}_{2 k} u^{k+1} u^{* k} \equiv-i u \Omega\left(u u^{*}\right),
$$

where $\Omega$ is an amplitude-dependent frequency. The quantity $u u^{*}$ is the nonlinear invariant of the motion, and the solution for $u$ is $\alpha=\rho e^{-i \psi}$, where $\rho=|u|$ is constant, and $\psi=\Omega t$. The nonlinear motion has been transformed into a phase-space rotation with an amplitude-dependent frequency. 
However, what is not obvious from the above discussion is that not all of the coefficients $T_{p q}$ in the normal form transformation, nor the tuneshift coefficients $\bar{U}_{2 k}$, are uniquely determined by demanding that the equation of motion for $u$ contain only resonant monomials. It is shown in Ref. [4] that the coefficients $T_{p q}$ for which $p=q+1$, i.e. $T_{21}, T_{32}$, etc., are not determined by this requirement. Such coefficients wiil be called "free terms" or "free functions". The above statements do not mean that the normal form is not affected by the free functions, but rather that some additional information must be supplied to fix their values. The criterion chosen for specifying the free functions is where the various methods of calculating normal forms differ from each other. A few possible choices will be described below.

The simplest choice is to put all the free functions to zero. This is perfectly valid, and reduces the computational effort required. Another possibility is to demand that the transformation to the normal form be a canonical transformation. In general, the relation between the Poisson Brackets $\left[z, z^{*}\right]$ and $\left[u, u^{*}\right]$ has the form

$$
\left[z, z^{*}\right]=\left[u, u^{*}\right]\left\{1+\sum_{k=1}^{\infty} \epsilon^{2 k} \tilde{P}_{2 k}\left(u u^{*}\right)^{k}\right\},
$$

when the equation of motion for $u$ has been brought into the normal form Eq. (6). Choosing the free functions to cancel all the $P_{2 k}$ makes the near-identity transformation in Eq. (3) a canonical transformation. This requires nonzero values for the free functions; it also leads to different values for the amplitude-dependent frequency shift terms $\tilde{U}_{2 k}$ in Eq. (6). The choice of canonical transformations is often automatic in the accelerator-physics literature, since there is a well-developed theory of such transformations. The present formulation displays the range of some of the other alternatives available for obtaining normal forms.

In this context, the minimal normal form method provides another prescription for choosing the values of the free functions. Instead of cancelling the Poisson Bracket terms $\widetilde{P}_{2 k}$, one chooses the free functions to cancel out the tuneshift corrections, the $\bar{U}_{2 k}$ 's. This turns out to be possible for all but the first term $\bar{U}_{2}$, i.e. the amplitudedependent frequency can be reduced to the form

$$
\Omega=\mu+i \epsilon^{2} \tilde{U}_{2} \rho^{2} \text {. }
$$

The reason is as follows. It is shown in Refs. [3] and [4] the free term of order 2k, viz. $T_{k+1 k}$, has no effect on the tuneshift term of the same order, $\bar{U}_{2 k}$, but it does affect the value of the next term $\tilde{U}_{2 k+2}$. The first term $\tilde{U}_{2}$ thus cannot be cancelled, but the others can, which therefore leaves us with Eq. (8).

Hence the minimal normal form method also leads to nonzero values for the free functions, but reduces the infinite series of higher order corrections to the normal form to only one term: this explains the name "minimal" normal form. As a result of this prescription, the exact solution for the normal form can be deduced at $O\left(\epsilon^{2}\right)$ already; the subsequent calculation consists only of determining the transformation between the original and new variables (the $T_{p q}$ coefficients) to higher and higher orders of $\epsilon$. The resulting transformation is not canonical: $\left[u, u^{*}\right]$ does not equal $\left[z, z^{*}\right]$. However, it is still symplectic: the value of the Poisson Bracket $\left[u, u^{*}\right]$ is constant in time, as is evident from Eq. (7). 
The method of minimal normal forms will now be extended to treat discrete maps, as opposed to differential equations in the previously published literature [3]. As explained above, such a reformulation is more suited to accelerator-based applications. Only a two-dimensional phase-space will be treated here; the extension to higher dimensions will be given later in this paper. We again define $z=x+i p$, where $(x, p)$ is the coordinate-momentum pair, and suppose that the map equation relating one turn to the next is

$$
z_{n+1}=\lambda z_{n}+\sum_{k=1}^{\infty} \epsilon^{k} Z_{k}\left(z_{n}, z_{n}^{*}\right),
$$

where $n$ is the turn number, $\lambda=e^{-i \mu}$, and the $Z_{k}$ are again homogenous polynomials of degree $k+1$ in $z$ and $z^{*}$ :

$$
Z_{k}=\sum_{p+q=k+1} Z_{p q} z^{p} z^{* q}
$$

The near-identity transformation to the normal form variable $u$ is given by

$$
z=u+\sum_{k=1}^{\infty} \epsilon^{k} T_{k}\left(u, u^{*}\right)
$$

with homogenous polynomials $T_{k}$ of degree $k+1$ in $u$ and $u^{*}$ :

$$
T_{k}=\sum_{p+q=k+1} T_{p q} u^{p} u^{* q}
$$

The $Z_{p q}$ are known; the $T_{p q}$ are to be determined. This form of the near-identity transformation is essentially the same as that of Scandale et al. [6], who express the

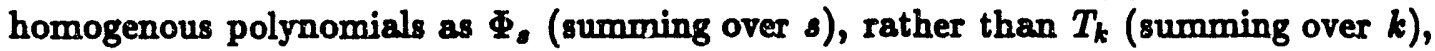
and is a nonlinear symplectic transformation. Only nonresonant normal forms will be treated in this paper, i.e. it will be supposed that $\mu /(2 \pi)$ is irrational, or $\lambda^{k} \neq 1$ for any $k \geq 1$. As before, the aim is to terminate the tuneshift corrections at a finite order. The equation of motion for $u$ assumes the form

$$
u_{n+1}=\lambda u_{n}+\sum_{k=1}^{\infty} \epsilon^{k} U_{k}\left(u_{n}, u_{n}^{*}\right),
$$

and the coefficients $T_{p q}$ are chosen so that the r.h.s contains only resonant mononaials $u^{k+1} u^{* k}$ :

$$
u_{n+1}=\lambda u_{n}\left[1+\sum_{k=1}^{\infty} \epsilon^{2 k} \frac{\bar{U}_{2 k}}{\lambda}\left(u u^{*}\right)^{k}\right] .
$$

This requirement again leaves undetermined the free functions, which are the $T_{p y}$ for which $p=q+1$. Analogous to the case of differential equations, the map for $u$ has the general form

$$
u_{n+1}=e^{-i \Omega\left(u_{n} u_{n}^{*}\right)} u_{n},
$$


where $\Omega$ consists of a sum of amplitude-dependent tuneshifts, and the minimal normal form procedure is to choose the free functions to terminate the series for $\Omega$ at a finite order. Unlike the case with differential equations, however, the terms in $\Omega$ are not simply the $\widetilde{U}_{2 k}$, hence one cannot terminate the series for the tuneshifts in $\Omega$ by setting the $\widetilde{U}_{2 k}$ to zero beyond $O\left(\epsilon^{2}\right)$. Instead the free function $T_{k+1 k}$ is chosen so that $\widetilde{U}_{2 k} / \lambda$ equals $\left(\widetilde{U}_{2} / \lambda\right)^{k} / k !$. This then yields the map

$$
\begin{aligned}
u_{n+1} & =\lambda u_{n}\left[1+\sum_{k} \frac{\epsilon^{2 k}}{k !}\left(\frac{\tilde{U}_{2 k}}{\lambda}\right)^{k}\left(u u^{*}\right)^{k}\right] \\
& =\lambda u_{n} \exp \left[\epsilon^{2}\left(\tilde{U}_{2} / \lambda\right) u u^{*}\right],
\end{aligned}
$$

or

$$
\Omega=\mu+i \epsilon^{2}\left(\tilde{U}_{2} / \lambda\right) \rho^{2},
$$

i.e., a single higher-order exponent. The nonlinear invariant of the motion is again $\rho^{2}=u_{n} u_{n}^{*}$. This is our generalization of Eq. (8) of the minimal normal form condition as applied to maps. As in the case of differential equations, setting all the free functions to zero would require less effort in calculating the $T_{p q}$ coefficients, but would yield an infinite series of amplitude-dependent tuneshift corrections

$$
\Omega=\mu+\epsilon^{2} \mu^{\prime} \rho^{2}+\epsilon^{4} \mu^{\prime \prime} \rho^{4}+\cdots,
$$

while a canonical transformation would yield both nonzero free functions and an infinite series of amplitude-dependent tuneshift corrections.

We note the following caveat: if the $O\left(\epsilon^{2}\right)$ tuneshift vanishes, so that the lowest order nonzero tuneshift is $\epsilon^{4} \mu^{\prime \prime}\left(u_{n} u_{n}^{*}\right)^{2}$, then the free functions should be chosen so that the map for $u_{n}$ has the form

$$
u_{n+1}=\lambda u_{n} \exp \left(-i \epsilon^{4} \mu^{\prime \prime} u_{n}^{2} u_{n}^{* 2}\right) .
$$

As a final development of the theoretical formalism in this paper, before turning our attention to numerical examples, the extension of the minimal normal form algorithm to higher phase-space dimensions will be given. We treat four dimensions in this section. Clearly, it is desirable for any such generalization to extend to six dimensions easily, so part of our effort will be to develop a suitable notation for the task.

At the first step, the linear part of the map is diagonalized. The details do not involve nonlinear dynamics, and will be omitted. The four eigenmodes will be denoted by $z_{1}, z_{1}^{*}, z_{2}$, and $z_{2}^{*}$. Only $z_{1}$ and $z_{2}$ need be treated below. In the absence of transverse $x-y$ coupling, $z_{1}=x+i p_{x}$ and $z_{2}=y+i p_{y}$, but such an identification is not required in the general formalism. We define $\lambda_{1}=e^{-i \mu_{1}}$ and $\lambda_{2}=e^{-i \mu_{2}}$ with an obvious notation. The equations of the map are then written in the form, using superscripts $i$ and $f$ for initial and final, respectively,

$$
z_{1}^{f}=\lambda_{1} z_{1}^{i}+\sum_{k=1}^{\infty} \epsilon^{k} Z_{k}^{(1)}\left(z_{1}^{i}, z_{1}^{i *}, z_{2}^{i}, z_{2}^{i *}\right)
$$




$$
z_{2}^{f}=\lambda_{2} z_{2}^{i}+\sum_{k=1}^{\infty} \epsilon^{k} Z_{k}^{(2)}\left(z_{1}^{i}, z_{1}^{i *}, z_{2}^{i}, z_{2}^{i *}\right) .
$$

We now introduce a vector index $\mathbf{m}=\left(m_{1}, m_{2}, m_{3}, m_{4}\right)$, and the norm $|\mathbf{m}|=\left|m_{1}\right|+$ $\cdots+\left|m_{4}\right|$. The above map equations can then be reexpressed as

$$
z_{j}^{f}=\lambda_{j} z_{j}^{i}+\sum_{\mathbf{m}} \epsilon^{|\mathbf{m}|-1} Z_{\mathbf{m}}^{(j)}\left|\mathbf{m}, z^{i}\right\rangle
$$

where $j=1$ or 2 , and

$$
|\mathrm{m}, z\rangle=z_{1}^{m_{1}} z_{1}^{* m_{2}} z_{2}^{m_{3}} z_{2}^{* m_{4}}
$$

is a shorthand notation to avoid a proliferation of symbols in the equations. The near-identity transformation to the normal form variables $u_{1}$ and $u_{2}$ is written as

$$
z_{j}=u_{j}+\sum_{\mathbf{m}} \epsilon^{|\mathbf{m}|-1} T_{\mathbf{m}}^{(j)}|\mathbf{m}, u\rangle
$$

where again $j=1,2$. Here $|\mathbf{m}, u\rangle$ is defined analogously to $|\mathrm{m}, z\rangle$ with $u$ in place of $z$. The above expressions are substituted into the map equations, and terms collected in powers of $u_{1}, u_{1}^{*}$, etc. The solutions for $u_{1}$ and $u_{2}$ are of the form $u_{j}=\rho_{j} e^{-i \psi_{j}}$, where $\rho_{1}^{2}=u_{1} u_{1}^{*}$ and $\rho_{2}^{2}=u_{2} u_{2}^{*}$ are the two nonlinear invariants of the motion.

The map equations for the normal form must now be specified. There is only one tuneshift parameter $\mu^{\prime}$ in two phase-space dimensions, but in four dimensions there are three:

$$
\left(\begin{array}{l}
\psi_{1}^{f} \\
\psi_{2}^{f}
\end{array}\right)=\left(\begin{array}{l}
\psi_{1}^{i} \\
\psi_{2}^{i}
\end{array}\right)+\left(\begin{array}{l}
\mu_{1} \\
\mu_{2}
\end{array}\right)+\epsilon^{2}\left(\begin{array}{ll}
\mu_{11} & \mu_{12} \\
\mu_{21} & \mu_{22}
\end{array}\right)\left(\begin{array}{l}
\rho_{1}^{2} \\
\rho_{2}^{2}
\end{array}\right),
$$

where $\mu_{12}=\mu_{21}$, i.e. the tuneshift parameter $\mu^{\prime}$ generalizes into a symmetric $2 \times 2$ matrix. Hence we seek equations of the form

$$
\begin{aligned}
& u_{1}^{f}=\lambda_{1} u_{1}^{i} \exp \left[-i \epsilon^{2}\left(\mu_{11} u_{1}^{i} u_{1}^{i *}+\mu_{12} u_{2}^{i} u_{2}^{i *}\right)\right], \\
& u_{2}^{f}=\lambda_{2} u_{2}^{i} \exp \left[-i \epsilon^{2}\left(\mu_{21} u_{1}^{i} u_{1}^{i *}+\mu_{22} u_{2}^{i} u_{2}^{i *}\right)\right] .
\end{aligned}
$$

Finally, the free terms must be identified. They are the coefficients of $u_{1}\left(u_{1} u_{1}^{*}\right)^{p}\left(u_{2} u_{2}^{*}\right)^{q}$ and $u_{2}\left(u_{1} u_{1}^{*}\right)^{p}\left(u_{2} u_{2}^{*}\right)^{q}$, respectively, i.e. $T_{p+1, p, q, q}^{(1)}$ and $T_{p, p, q+1, q}^{(2)}$ where $p, q=0,1, \ldots$ with $p+q \geq 1$. The free terms are obviously chosen 80 as to attain the tuneshift formula Eq. (24).

The formalism for six phase-space dimensions is formally exactly the same as in four dimensions. The index $m$ is extended to six components, and the tuneshift formula to

$$
\left(\begin{array}{l}
\psi_{1}^{f} \\
\psi_{2}^{f} \\
\psi_{3}^{f}
\end{array}\right)=\left(\begin{array}{l}
\psi_{1}^{i} \\
\psi_{2}^{i} \\
\psi_{3}^{i}
\end{array}\right)+\left(\begin{array}{l}
\mu_{1} \\
\mu_{2} \\
\mu_{3}
\end{array}\right)+\epsilon^{2}\left(\begin{array}{lll}
\mu_{11} & \mu_{12} & \mu_{13} \\
\mu_{21} & \mu_{22} & \mu_{23} \\
\mu_{31} & \mu_{32} & \mu_{33}
\end{array}\right)\left(\begin{array}{l}
\rho_{1}^{2} \\
\rho_{2}^{2} \\
\rho_{3}^{2}
\end{array}\right),
$$

where the tuneshift matrix is again symmetric: $\mu_{i j}=\mu_{j i}$, and the nonlinear invariants are $\rho_{j}^{2}=u_{j} u_{j}^{*}, j=1,2,3$. More complete mathematical details of the formalism for rour and six phase-space dimensions are given in Ref. [4]. 


\section{NUMERICAL RESULTS}

The numerical studies below will be restricted to two phase-space dimensions. Results for higher phase-space dimensions, though important, are beyond the scope of this paper. We first study some differential equations, then maps. The first model is that of a Duffing oscillator

$$
\dot{z}=-i \mu z+\frac{i \epsilon^{2}}{8}\left(z+z^{*}\right)^{3},
$$

which can be derived from the more familiar equations

$$
\begin{aligned}
& \dot{x}=\mu p, \\
& \dot{p}=-\mu x+\epsilon^{2} x^{3} .
\end{aligned}
$$

We may set $\mu=1$ without loss of generality. Three methods of solution were used: (1) set all free functions to zero (the " $F=0$ " choice), (2) a canonical transformation (denoted "CT" below), and (3) the minimal normal form (denoted "MNF"). For this perturbation, the $O\left(\epsilon^{2}\right)$ tuneshift parameter can be read off immediately and is

$$
\mu^{\prime}=-\frac{3}{8},
$$

for all of the above methods. It is only at higher orders that the tuneshift parameters, etc., differ between the methods.

To examine the convergence of the series expansions of the various methods above, D'Alembert's test of convergence [7] suggests that we plot the magnitudes of the $k^{\text {th }}$ order terms $\left|T_{k}\right|$, or $\log \left|T_{k}\right|$, as a function of $k$. The function actually plotted was $\log \left(\sum_{i+j=k+1}\left|T_{i j}\right|\right)$, i.e. the sum of the magnitudes of the coefficients of the individual terms in each $T_{k}$. The results are shown in Fig. 1, where the curves corresponding to the various choices are labelled $F=0, C T$, or MNF, respectively. It is striking that the coefficients from the first two methods $(F=0$ and $C T)$ do not decrease with the order at all, whereas those calculsted using the MNF decrease rapidly. This is equivalent to saying that the minimal normal form achieves, at low orders of perturbation theory, a better approximation to the exact solution, thus requiring smaller higher order corrections.

Another important test of the accuracy of the various perturbative solutions is to compute the value of the energy. Since the equation of motion is autonomous, the Hamiltonian is a first integral of the motion, and the energy should remain constant as a function of time. In general, the value of any first integral of the motion should be computed and checked for constancy as a function of time. For the Duffing oscillator of Eq. (27), the energy is

$$
E=\frac{\mu}{2}\left(p^{2}+x^{2}\right)-\frac{\epsilon^{2} x^{4}}{4}
$$

In our analysis, $\mu=\epsilon=1$. The amplitude was set at $\rho \simeq 0.5$, so as to be well within the separatrix, so that all the methods would converge rapidly. (The unstable fixed 


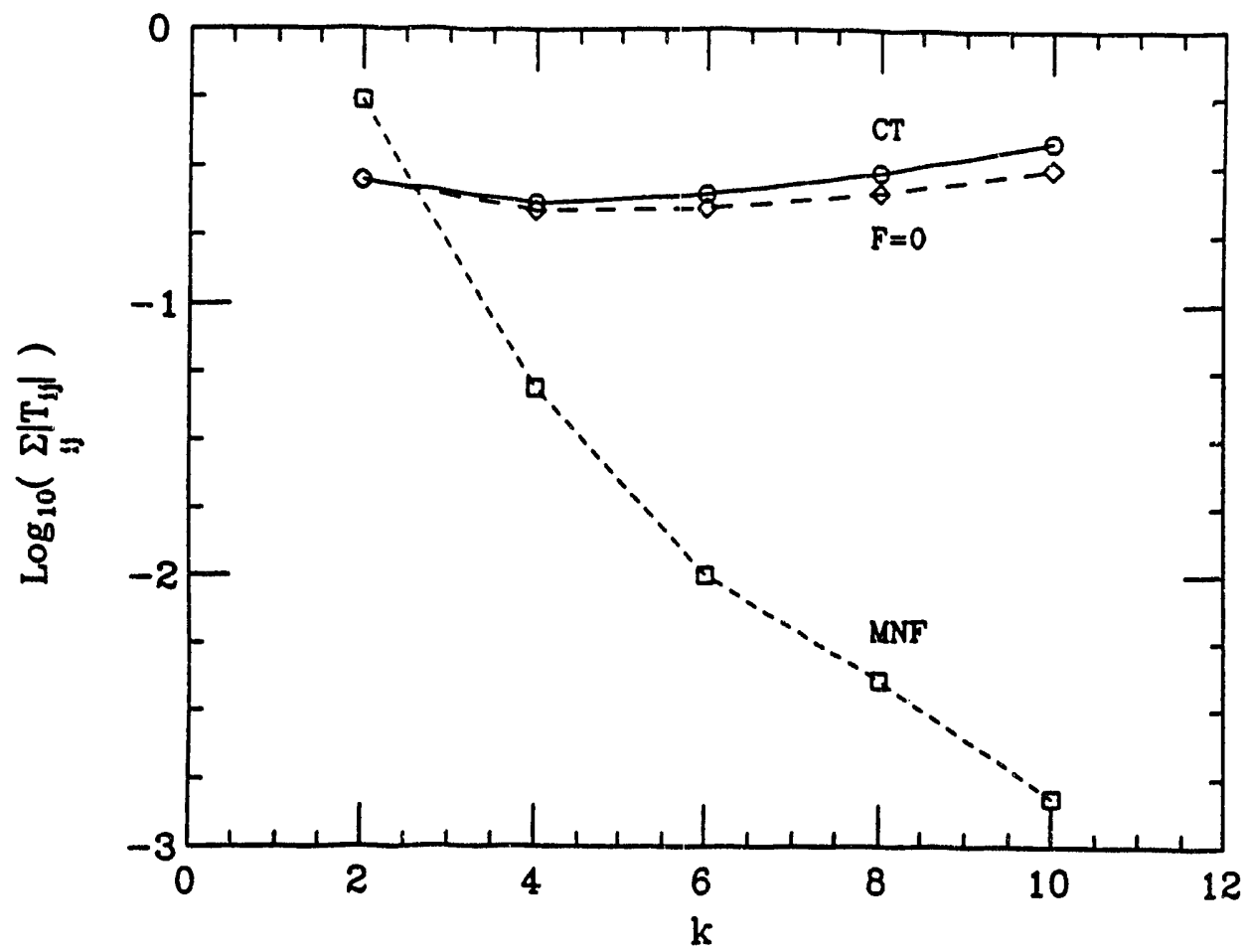

Figure 1: Graph of the magnitudes of the coefficients in the normal form transformation as a function of the order of perturbation theory, for various methods of choosing the free functions for a Duffing oscillator.

points are at $(x, p)=( \pm 1,0)$, i.e. $z= \pm 1$.) For each method, the calculations were performed to $O\left(\epsilon^{4}\right)$ and $O\left(\epsilon^{6}\right)$, i.e. the near-identity transformation to the normal form was calculated through $T_{4}$ or $T_{6}$, respectively, and the tuneshifts were also calculated through $O\left(\epsilon^{4}\right)$ or $O\left(\epsilon^{6}\right)$, for the $F=0$ and CT cases. For the MNF, the tuneshift terminated at $O\left(\epsilon^{2}\right)$, of course. The value of the relative error $\left(E-E_{0}\right) / E_{0}$, where $E_{0}$ is the initial energy, is plotted against the time in Fig. 2. A total of six curves are shown and labelled, viz. dashes for the MNF, solid for the CT, and crosses for the $F=0$ choice. The results for the $F=0$ and CT methods are very similar, but that for the MNF is quite clearly better than both. Even at $O\left(\epsilon^{4}\right)$, the MNF yields an error comparable to that from the others at $O\left(\epsilon^{6}\right)$, while when the MNF is used to $O\left(\epsilon^{6}\right)$ the error is almost invisible on the scale of Fig. 2.

On the above evidence, the minimal normal form appears to be a good choice when utilizing the freedom in setting up the near-identity transformation to the normal form, but more evidence is required, using other equations of motion. Use of a quadratic perturbation, viz.

$$
\dot{z}=-i \mu z+\frac{i \epsilon^{2}}{8}\left(z+z^{*}\right)^{3},
$$




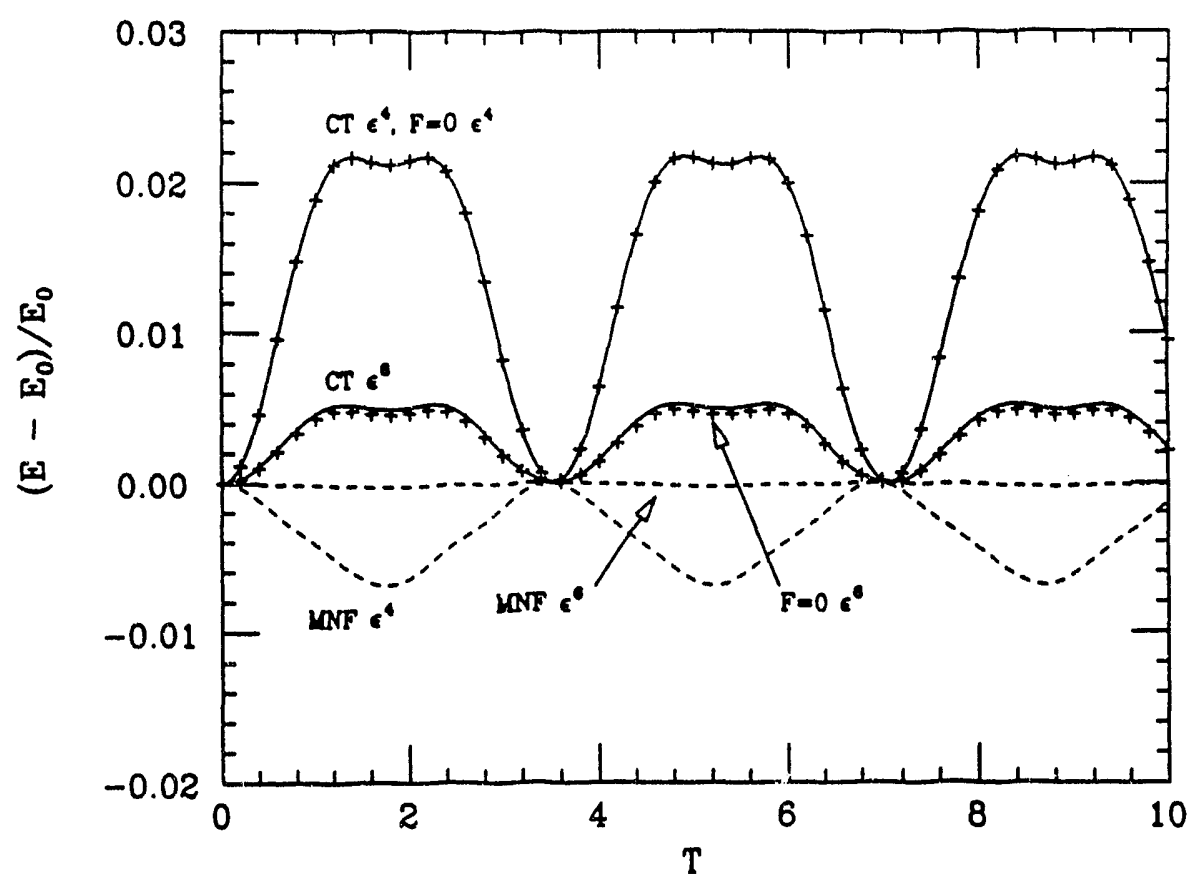

Figure 2: Graph of the relative error $\left(E-E_{0}\right) / E_{0}$ in the energy as a function of time, for calculations through fourth and sixth order, for various methods of performing the normal form transformation. The equation of motion contained a cubic nonlinear term.

yields results even more impressive than those from the Duffing oscillator. Thus, fur two well-known models, the minimal normal form method yields better results than the more traditional techniques. However, there are cases where the use of the minimal normal form as described above is not so effective. Suppose one uses a combination of quadratic and cubic perturbations, e.g. a potential

$$
V=-\frac{8}{25} \epsilon x^{3}+\frac{\epsilon^{2} x^{4}}{4}
$$

with $\mu=\epsilon=1$, for which the $O\left(\epsilon^{2}\right)$ tuneshift parameter is $\mu^{\prime} \simeq-0.009$, i.e. almost zero. The minimal normal form method works badly in this case. The corresponding graph to Fig. 1 is shown in Fig. 3. The MNF coefficients, this time, are larger than those from the $F=0$ and CT methods. This behavior is explained in Ref. [4], where it is shown that, to achieve the MNF criterion, the free functions are inversely proportional to $\mu^{\prime}$, and hence become large if $\mu^{\prime}$ is small. The coefficients in the $F=0$ and CT methods are not directly dependent on the value of $\mu^{\prime}$, hence their behavior is not much different between Figs. 1 and 3.

However, it was shown in Ref. [4] how to cure this problem. As pointed out above, if $\mu^{\prime}=0$, then the MNF prescription should be modified to retain the $O\left(\epsilon^{4}\right)$ tuneshift 


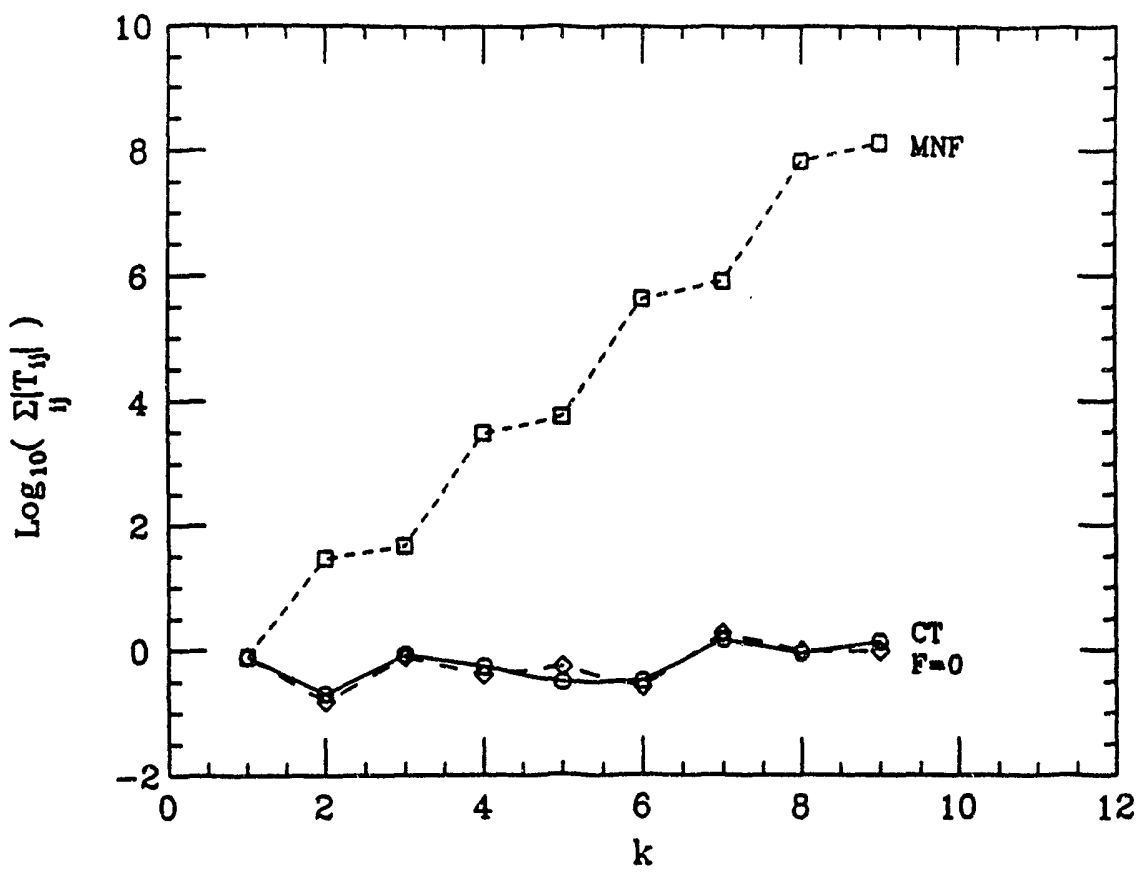

Figure 3: Graph of the magnitudes of the coefficients in the normal form transformation as a function of the order of perturbation theory, with quadratic and cubic nonlinear terms chosen so as to yield a small second order tuneshift parameter.

parameter $\mu^{\prime \prime}$, and to use the free functions to achieve an tuneshift of $\epsilon^{4} \mu^{\prime \prime} \rho^{2}$ in the normal form. The free functions will then be inversely proportional to $\mu^{\prime \prime}$. Using the free functions in this way, the magnitudes of the coefficients in the series expansion are shown in Fig. 4, together with the and CT methods, which are unchanged from Fig. 3. Strictly speaking, the MNF prescription was only applied approximately, i.e. the tuneshift coefficients beyond $\mu^{\prime \prime}$ were made small but not exactly zero, because the equations to be solved were more complicated and beyond the scope of the present formalism. The MNF coefficients are now an order of magnitude smaller than those from the $F=0$ and CT methods, instead of eight orders of magnitude larger as in Fig. 3. The improvement in the MNF results is dramatic.

Hence we may conclude that the minimal normal form has the potential to be a good method of exploiting the freedom available in the transformation from the original phase-space coordinates to the normal form. It holds out the promise of offering a good approximation to the exact solution using only low orders of perturbation theory, thereby requiring smaller higher order corrections than other methods such as a canonical transformation, but there are some caveats to the implementation of the method, as noted above, if the magnitude of the lowest order tuneshift parameter $\mu^{\prime}$ was small, or zero. The merit of the minimal normal form method for discrete maps 


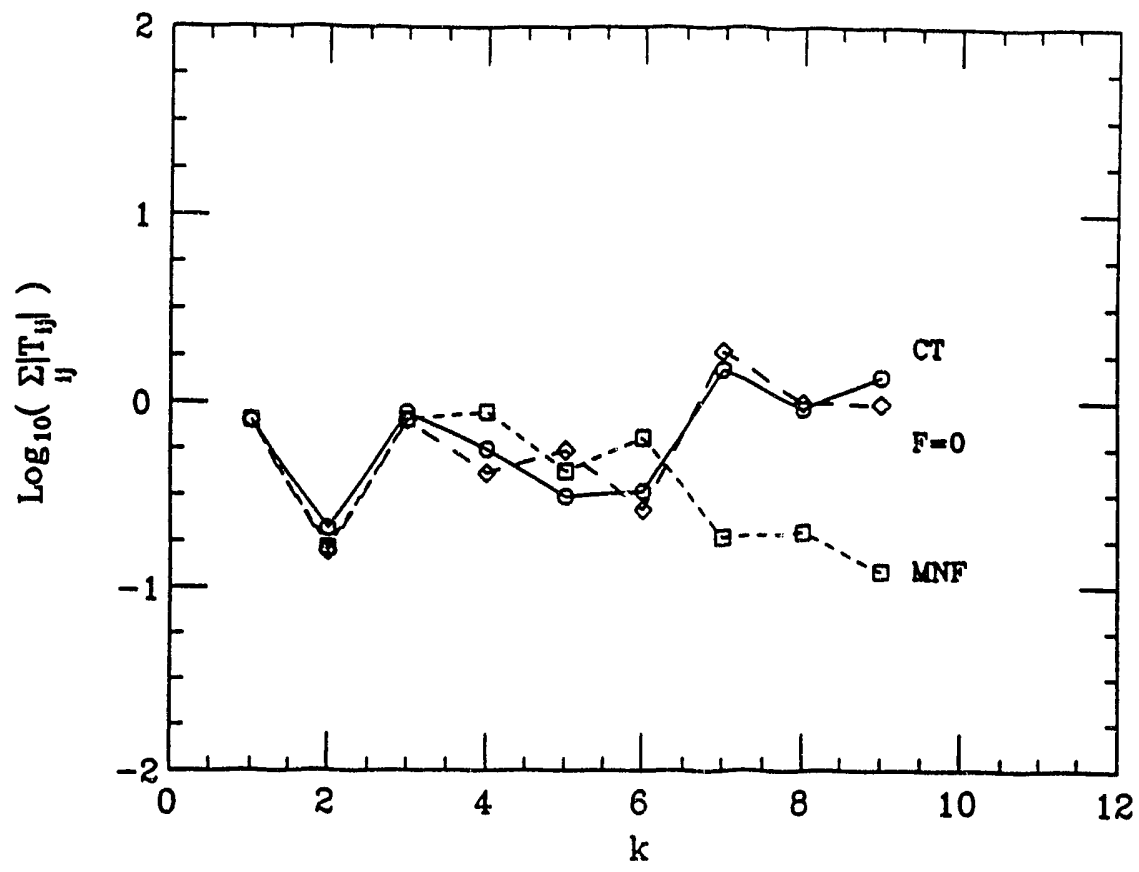

Figure 4: Graph of the magnitudes of the coefficients in the normal form transformation as a function of the order of perturbation theory. Both the second and fourth order tuneshift parameters were retained in the minimal normal form method.

will now be investigated.

The calculations of the expressions for the normal form for discrete maps are similar to those for differential equations. The three choices for the norma urm will again be denoted $F=0, C T$ and MNF. The maps treated below used a single thin-lens sextupole or octupole, i.e. a quadratic or cubic nonlinearity. A plot of the phasespace trajectories for these maps is shown in Fig. 5. The tunes were set to $\nu=0.255$ and $\nu=0.34$ respectively, where $\mu=2 \pi \nu$, and the value of $\epsilon$ was chosen so that the separatrices would be at $x^{2}+p^{2} \simeq 1$ in both cases. This leads to the choices $\epsilon=0.2$ and 0.1 , respectively. For the octupole, the map equation is

$$
z_{n+1}=\lambda z_{n}+\frac{i \epsilon^{2} \lambda}{8}\left(z_{n}+z_{n}^{*}\right)^{3} \text {. }
$$

The sextupole map would normally be very similar

$$
z_{n+1}=\lambda z_{n}+\frac{i \epsilon \lambda}{4}\left(z_{n}+z_{n}^{*}\right)^{2},
$$

i.e. the Hénon map [8]. For aesthetic reasons, however, to create phase-space trajectories symmetric around the $x$ axis, the sextupole was located diametrically opposite the observation point, leading to the map equation 


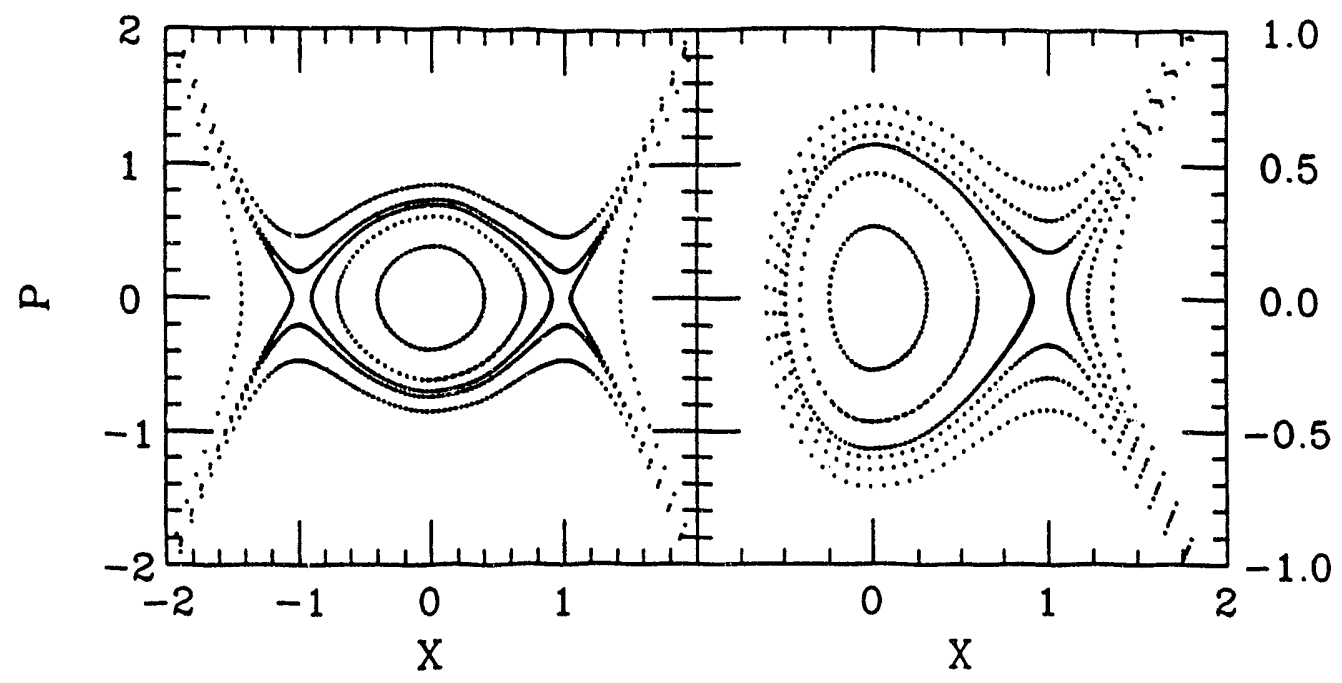

Figure 5: Phase-space trajectories for mups with a thin-lens octupole (left) and sextupole (right). The tunes are abolled in each figure. The nonlinear multipole strength was chosen to place the sef trix at $x^{2}+p^{2} \simeq 1$ in each case.

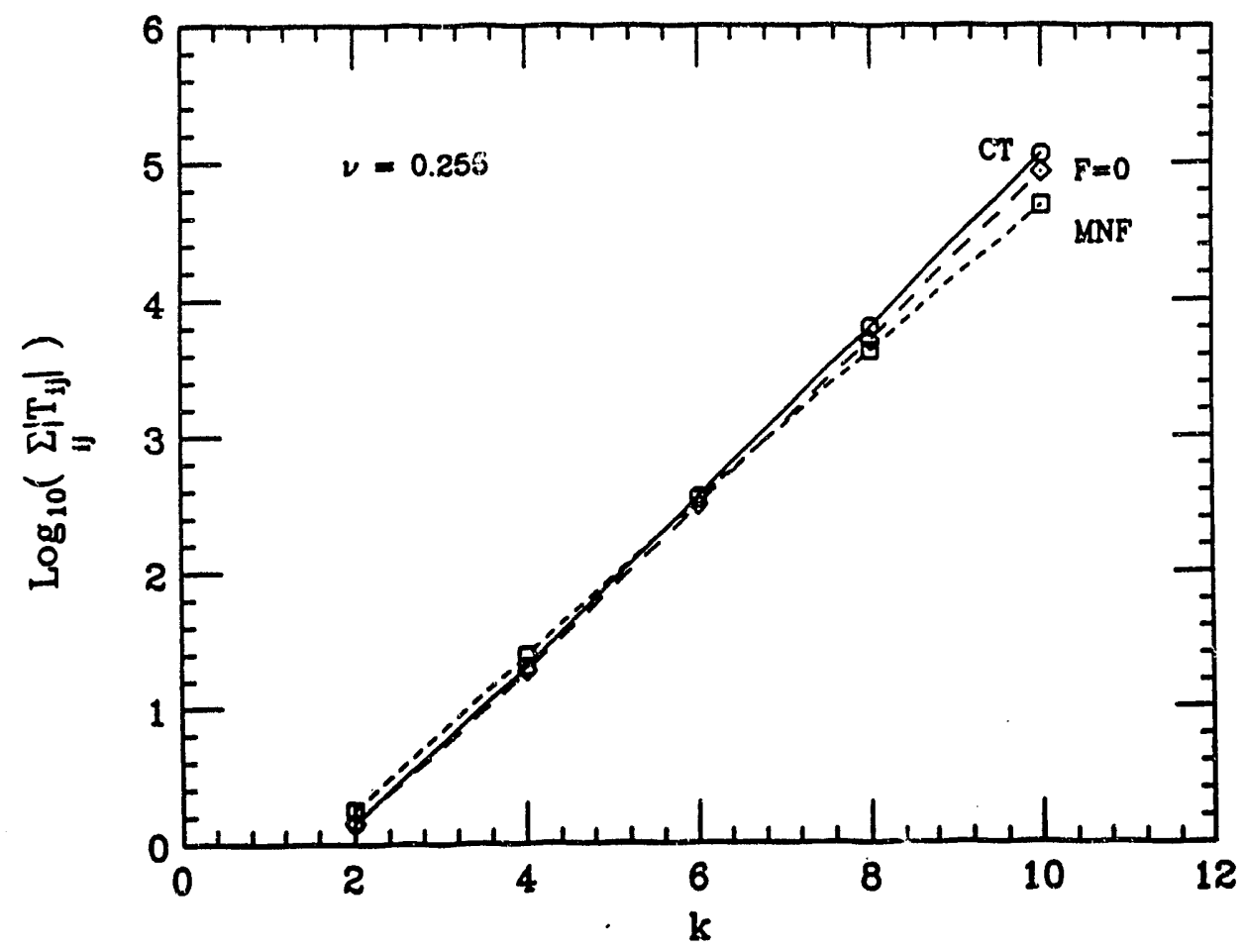

Figure 6: Graph of the magnitudes of the coefficients in the normal form transformation as a function of the order of perturbation theory, for various methods of choosing the free functions, for a map with a single thin-lens octupole, using a tune of $\nu=0.255$. 


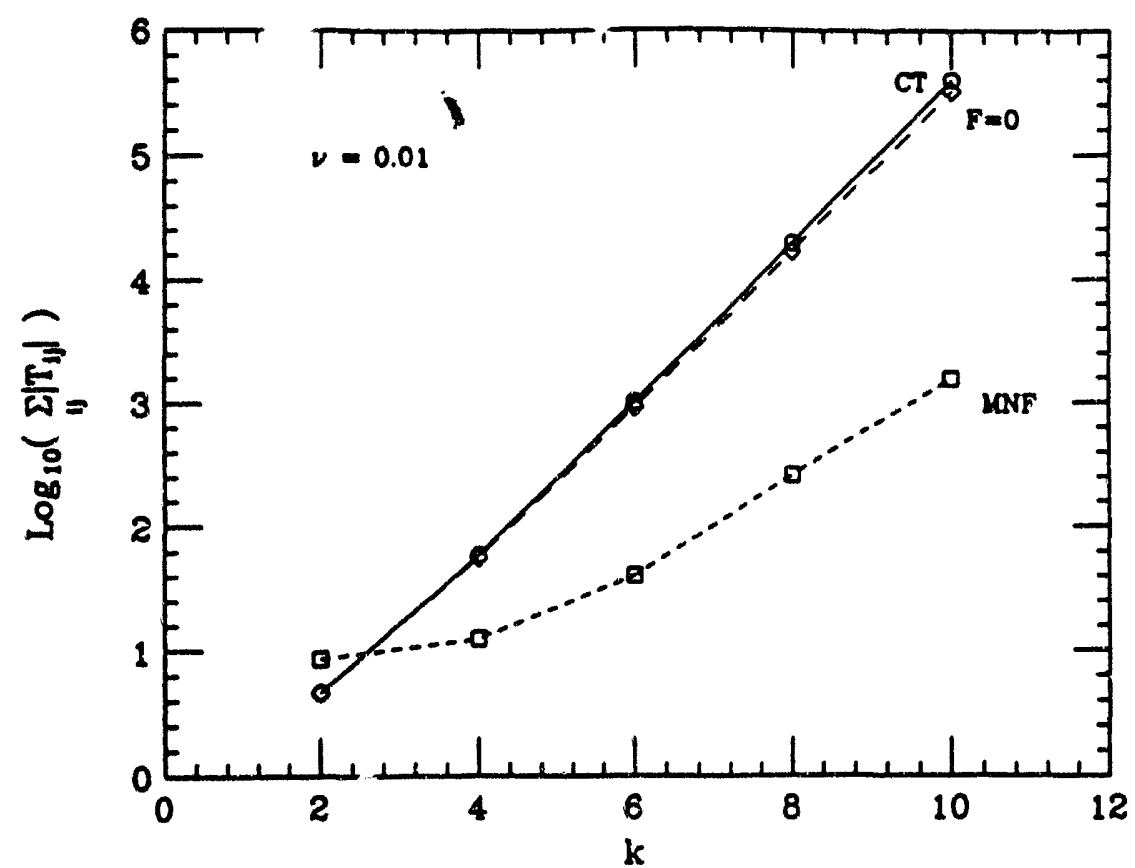

Figure 7: Sane as the previous figure, but with a tune of $\nu=0.01$.

$$
z_{n+1}=\lambda z_{n}+\frac{i \epsilon \sqrt{\lambda}}{4}\left(\sqrt{\lambda} z_{n}+\sqrt{\lambda^{*}} z_{n}^{*}\right)^{2}
$$

which was used to generate Fig. 5. It has been verified that the location of the sertupole made no difference to the convergence of the series expansion for the normal form. The values of the $O\left(\epsilon^{2}\right)$ tuneshift parameter are

$$
\mu^{\prime}=-\frac{3}{8},
$$

for the thin-lens octupole kick in Eq. (33), and

$$
\mu^{\prime}=\frac{i}{8} \frac{2 \lambda^{3}+3 \lambda^{2}+3 \lambda+2}{\lambda^{3}-1},
$$

for the thin-lens sextupole kick in Eq. (35).

As explained above, in accordance with D'Alembert's test of convergence [7], the various methods for calculating the normal form were compared by plotting the function $\log \left(\sum_{i+j=k+1}\left|T_{i j}\right|\right)$ against the order $k$. The results for the thin-lens octupole map Eq. (33), with a tune of $\nu=0.255$, are shown in Fig. 6. The results from all three methods are almost the same, and although the MNF result is smaller than the others, the difference is slight. It is not immediately clear why this is so; perhaps it may be due to the presence of a small denominator in the coefficients, caused by the location of large low-order resonance islands close to the origin. 


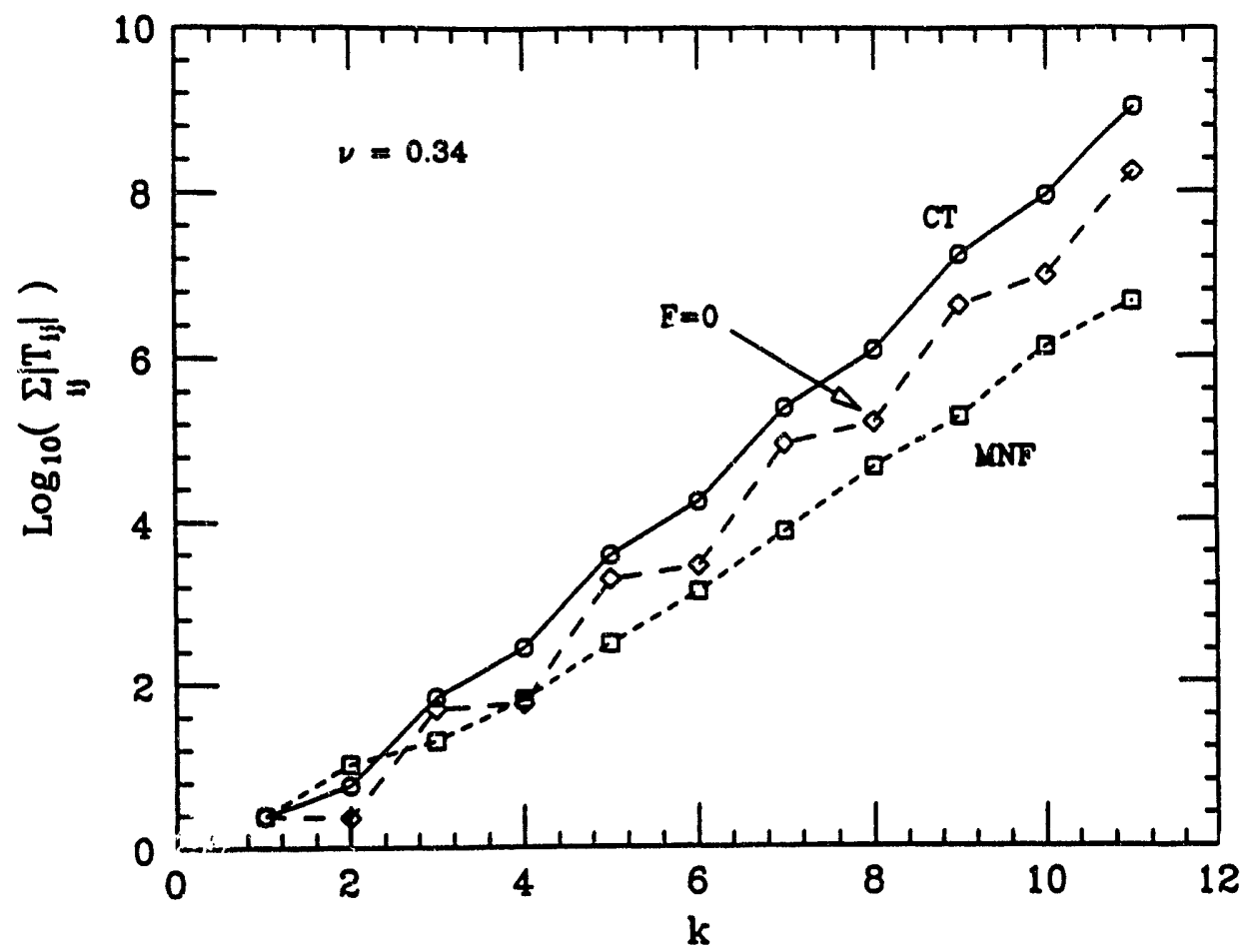

Figure 8: Graph of the magnitudes of the coefficients in the normal form transformation as a function of the order of perturbation theory, for various methods of choosing the free functions, for a map with a single thin-lens se xtupole. The small-amplitude tune was $\nu=0.34$.

In an attempt to approximate the conditions of the differential equation, the calculation was repeated using a tune of $\nu=0.01$, i.e. a small phase advance per turn. The results are shown in Fig. 7. The MNF result, this time, is distinctly better than the others, the coefficients being smaller by two orders of magnitude at the tenth order.

To examine the behavior for a different map, the thin-lens sextupole map of Eq. (35) was studied next. The results are shown in Fig. 8, for a tune of $\nu=0.34$. This time, the MNF coefficients for the normal form are clearly smaller than those from the $F=0$ and CT methods. Use of a combined sextupole and octupole perturbation did nat make any significant difference to the above conclusions.

Wence the minimal normal form method, when applied to discrete maps, sometimes yivids better results than other methods, but the difference is not as pronounced as when solving differential equations. In all of the maps studied above, the free functions were chosen to cancel the higher order tuneshift coefficients beyond $O\left(\epsilon^{2}\right)$. However, in those cases where the minimal normal form results were not better than those from other methods, it may be a good idea to retain both the $O\left(\epsilon^{2}\right)$ and $O\left(\epsilon^{4}\right)$ tuneshift terms, or to extend the method to treat resonant normal forms (motivated by the presence of large resonance islands in the phase-space of the octupole map). This requires a more sophisticated formalism and computer program, because the equations to be solved are not as straightforward. Future reports will address the issue. 


\section{CONCLUSION}

The method of normal forms has been reviewed and the superiority of the minimal normal form method has been demonstrated for ordinary nonlinear autonomous differential equations. The minimal normal form method has also been extended, to treat discrete maps. The application to the evaluation of one-turn maps for accelerators yields mixed results, hence the superiority of the minimal normal form is not as clearly visible. Further studies in investigating the effect of small denominators in the vicinity of low order resonances will be attempted, to improve the convergence of the perturbation expansion, and numerical model evaluations will be carried out for higher dimensional phase space.

A major application of the method presented here in the calculation of the one-turn map is to use the map to evaluate the effect of nonlinear perturbations on the lattice functions, such as betatron functions, the dispersion function, chromaticity, and tunes of the acrelerator. If such improvements are possible, the minimal normal form method can serve as a standard way of calculating lattice functions of an accelerator or storage ring with small nonlinear imperfections.

\section{ACKNOWLEDGEMENTS}

The authors would like to thank Professors P. Kahn and Y. Zarmi for fruitful discussiuns on the method of minimal normal forms, and Mrs. D. Murray for help on part of the calculations. Discussions with D. Abell, P. Channel, J. Ellison, E. Forest, and $A$. Sessler are also appreciated.

\section{REFERENCES}

1. E.D. Courant and H.S. Snyder, Ann. Phys., 3, 1 (1958).

2. E. Forest and K. Hirata, KEK Report 92-12, (1992). See also references therein.

3. P.B. Kahn and Y. Zarmi, Physica D, 54, 65, (1991).

4. S.R. Mane and W.T. Weng, Brookhaven National Laboratory Report BNL-48249 (Nov. 1992), and submitted to Phys. Rev. E.

5. G. Turchetti, Nonlinear Problems in Future Particle Accelerators (World Scientific, Singapore, 1991), eds. W. Scandale and G. Turchetti, p. 16.

6. W.Scandale, F. Schmidt and E. Todesco, Part. Accel., 35, 53 (1991).

7. I. S. Gradshteyn and I. M. Rhyzik, Table of Integrals, Series, and Products (Academic Press, Inc., New York, 1980), 4th ed., p. 5.

8. M. Hénon, Chsotic Behavior of Deterministic Systems, (North-Holland, New York, (1983)) eds. G. Iooss et al., p. 53.

This report was prepared as an account of work sponsored by an agency of the United States Government. Neither the United States Government nor any agency thereof, nor any of their employees, makes any warranty, express or implied, or assumes any legal liability or responsibility for the accuracy, completeness, or usefulness of any information, apparatus, product, or process disclosed, or represents that its use would not infringe privately owned rights. Reference herein to any specific commercial product, process, or service by trade name, trademark, manufacturer, or otherwise does not necessarily constitute or imply its endorsement, recommendation, or favoring by the United States Government or any agency thereof. The views and opinions of authors expressed herein do not necessarily state or reflect those of the United States Government or any agency thereof. 

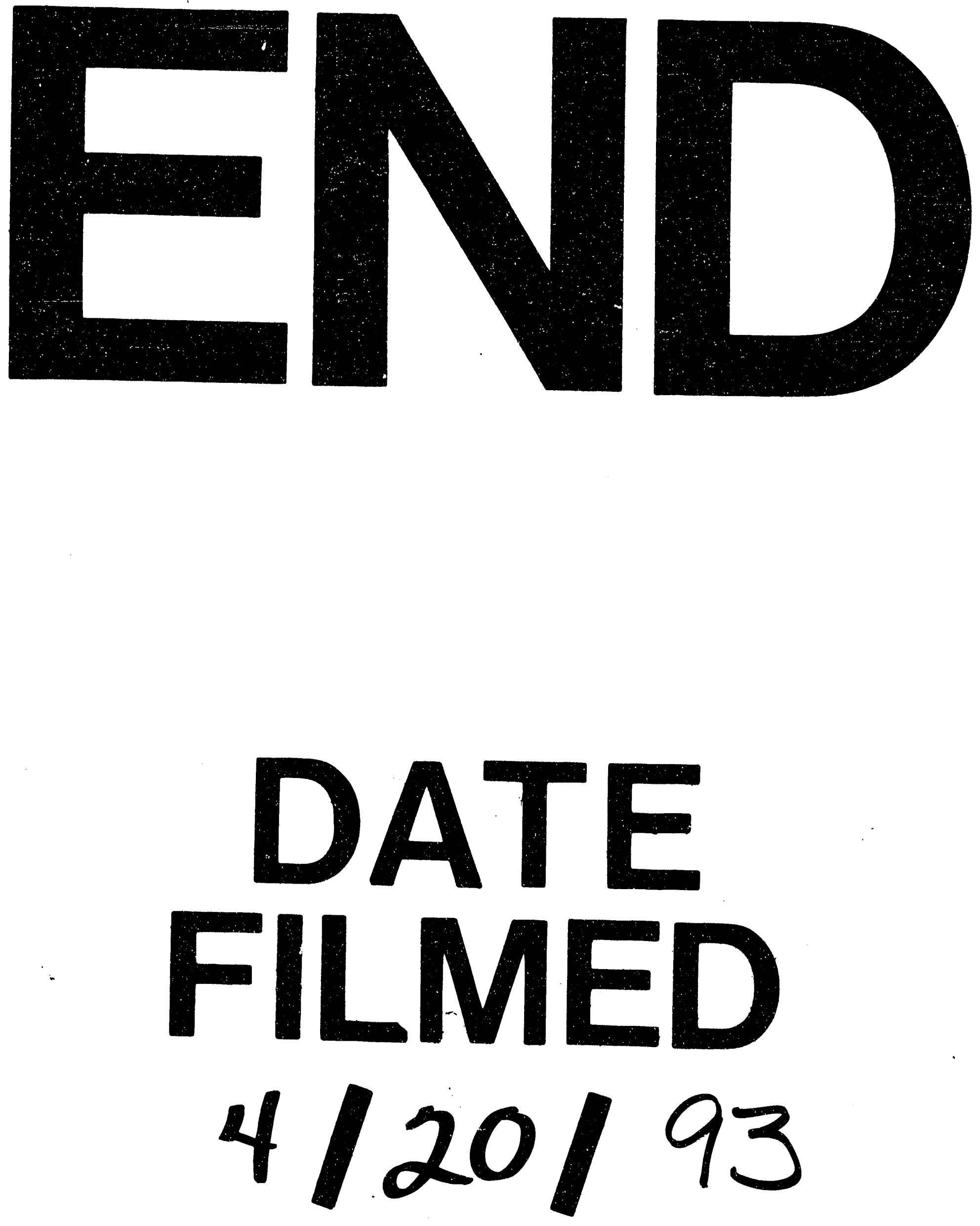
